\title{
Optical Sensing Based on Light Coupling Between Two Parallel Long-Period Fiber Gratings
}

\author{
Kin Seng CHIANG ${ }^{1,2}$, Yunqi LIU ${ }^{1,3}$, Qing LIU $^{1}$, and Yunjiang RAO ${ }^{2}$ \\ ${ }^{1}$ Department of Electronic Engineering, City University of Hong Kong, 83 Tat Chee Avenue, Hong Kong, China \\ ${ }^{2}$ Key Laboratory of Optical Fiber Sensing \& Communications (Ministry of Education), University of Electronic Science \\ and Technology of China, Chengdu, 611731, China \\ ${ }^{3}$ Key Lab of Special Fiber Optics and Optical Access Networks, School of Communication and Information Engineering, \\ Shanghai University, Shanghai, 200072, China \\ *Corresponding author: Kin Seng CHIANGＥ-mail: eeksc@cityu.edu.hk
}

\begin{abstract}
A new optical intensity-based sensing mechanism for the measurement of refractive index and minute displacement is proposed in this paper, which is based on modifying the amount of light coupled between two parallel long-period fiber gratings. The characteristics of this sensing mechanism with experiments and simulation results are demonstrated.
\end{abstract}

Keywords: Long-period fiber grating, optical fiber sensor, refractive-index sensor, displacement sensor, intensity-based sensor

\section{Introduction}

Long-period fiber grating (LPFG) formed in a single-mode fiber, which enables strong light coupling from the guided core mode to selected cladding modes at specific resonance wavelengths [1], has found many applications in optical communications and sensing $[2,3]$. By putting a LPFG in close proximity to another identical LPFG, light at the resonance wavelength can be coupled effectively between two parallel fibers [3, 4]. This light-coupling mechanism has been demonstrated as a principle for the realization of 4-port [3-8] and 6-port $[9,10]$ broadband couplers and add/drop multiplexers for application in coarse wavelength division multiplexing (CWDM) systems. By using two parallel gratings written in $\mathrm{B}-\mathrm{Ge}$ co-doped fibers by a $\mathrm{CO}_{2}$ laser, we have achieved a peak coupling efficiency of $86 \%$ [8], which is the highest value reported so far. One may refer to [11] for a comprehensive review of the development of long-period gating coupling devices, including a discussion of the implementation of such devices with planar optical waveguides. In this paper, we show that the principle of light coupling between two LPFGs can be explored as an effective mechanism for sensing refractive index and displacement.

The principle of light coupling between two parallel LPFGs has been analyzed in detail [5]. The grating in the input fiber couples light from the guided core mode to the cladding mode of the fiber. At the same time, the cladding mode of the parallel fiber is excited through evanescent-field coupling between two fibers. The grating in the parallel fiber couples the cladding mode to the guided core mode. The light rejected from the input fiber is collected by the parallel fiber, so the output spectra of two fibers

Received: 3 August 2010 / Revised version: 30 August 2010

C The Author(s) 2010.This article is published with open access at Springerlink.com 
are complementary to each other. The efficiency of the whole process depends critically on the efficiency of evanescent-field coupling between the cladding modes, which depends on the spatial overlap of the cladding-mode fields of two fibers. Therefore, the power of the output light from the two fibers depends on the surrounding refractive index, the offset distance between two gratings, and, most sensitively, to the separation between the two fibers. This paper presents our first results on the sensing characteristics of two parallel LPFGs.

\section{Refractive-index sensing}

Figure 1 shows the experimental setup for the demonstration of the operation principle of the sensor formed with two parallel LPFGs. Light from a commercial $(\mathrm{C}+\mathrm{L})$-band amplified spontaneous emission (ASE) source is launched into one fiber and the output light of the other fiber is measured with a broadband photodetector (PD2) as the sensor output. In practice, the input light could be tapped with a 10:90 fiber coupler and measured with another photodetector (PD 1) to provide intensity reference.

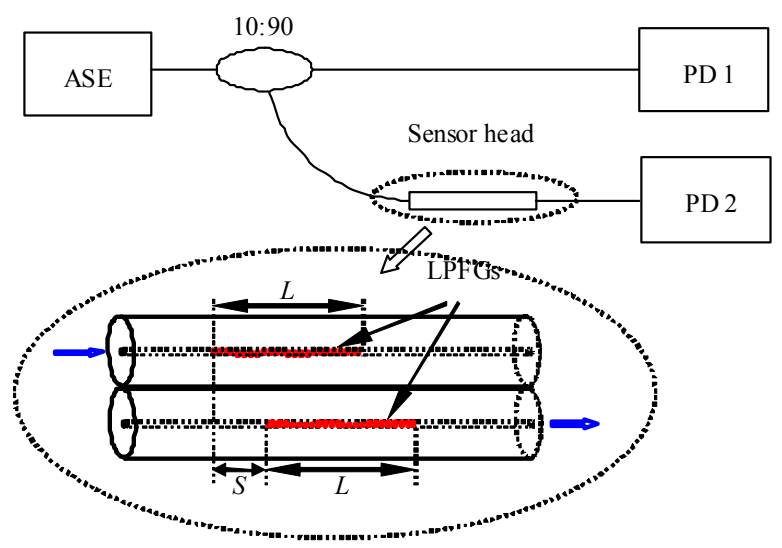

Fig. 1 Experimental setup for the characterization of two parallel LPFGs for refractive-index sensing, where light is launched into one fiber and detected from the other fiber as the sensor output and the two gratings with identical length $L$ are allowed to offset by a distance $S$.

In the first experiment, we used two LPFGs written in a B-Ge co-doped single-mode fiber (Fibercore PS1250/1500) by $\mathrm{CO}_{2}$ laser irradiation [8]. The gratings had a period of $345 \mu \mathrm{m}$ and a length of $34.5 \mathrm{~mm}$ (100 periods). The transmission spectra of the two gratings show clean rejection dips at $1578.8 \mathrm{~nm}$ and $1578.5 \mathrm{~nm}$, respectively, which corresponded to the coupling to $\mathrm{LP}_{08}$ mode. The contrasts and 3-dB bandwidths of the rejection bands of two gratings were $23 \mathrm{~dB}$ and $28 \mathrm{~dB}$, and $29 \mathrm{~nm}$ and $27 \mathrm{~nm}$, respectively. All were measured in air.

The coupling efficiency between two fibers can be varied by controlling the offset distance between the two gratings [5]. In the experiment, the offset distance was fixed at $10 \mathrm{~mm}$ to provide sufficiently strong coupling. Figure 2 shows the dependence of the measured output power on the surrounding refractive index. As shown in Fig. 2, the output power from the parallel fiber increases with the surrounding refractive index up to a point when the surrounding refractive index becomes close to the refractive index of the fiber cladding. The variation of the output power with the surrounding refractive index is consistent with the trend of the evanescent-field coupling coefficient [5].

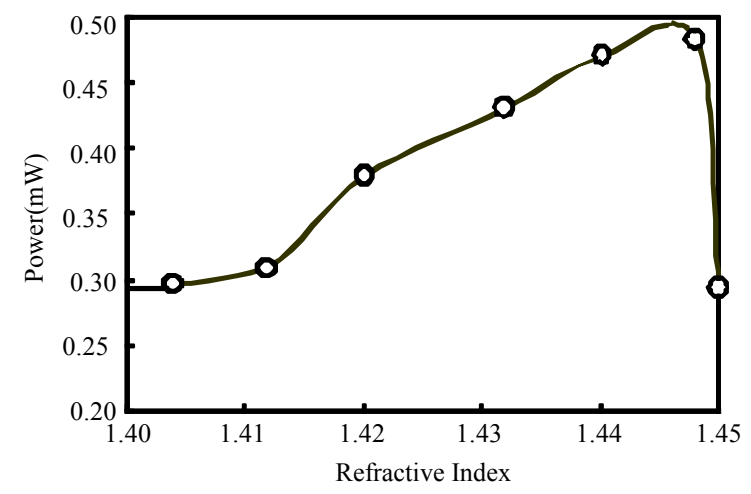

Fig. 2 Dependence of the sensor output on the surrounding refractive index for two parallel $\mathrm{CO}_{2}$-laser written LPFGs with an offset distance of $10 \mathrm{~mm}$.

The simulation results for refractive-index sensing obtained by solving a set of coupled-mode equations [5], are shown in Fig. 3 for a fiber with a core index of 1.4490 , a core radius of $3.78 \mu \mathrm{m}$, a cladding index of 1.4443 , and a cladding radius of 
$62.4 \mu \mathrm{m}$. The grating parameters used in the simulation are the same as those used in the experiments. The spectral power density of the light source is assumed to be $50 \mu \mathrm{W} / \mathrm{nm}$. The simulation results agree qualitatively with the experimental data.

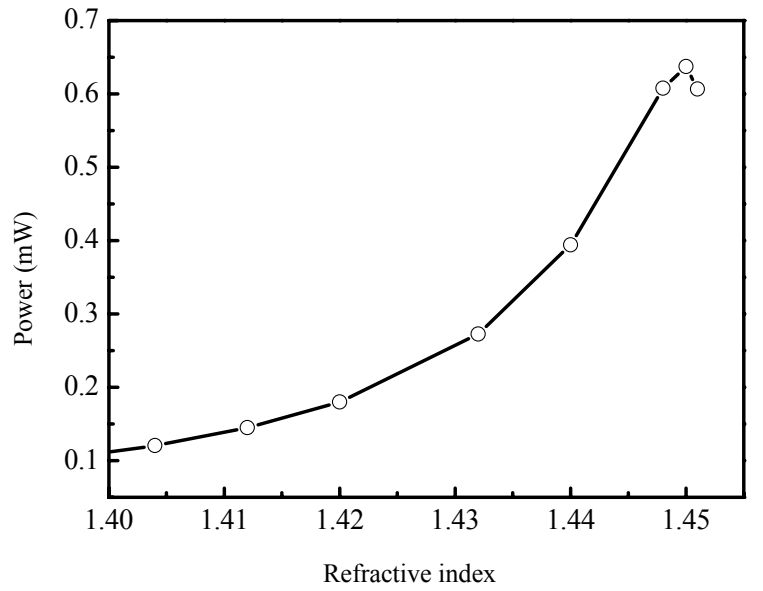

Fig. 3 Simulation results for refractive-index sensing with two parallel LPFGs.

In the second experiment, we used LPFGs written in the same B-Ge co-doped fiber by ultra-violet (UV) irradiation (at $248 \mathrm{~nm}$ ) through an amplitude mask. The gratings were 32-mm long and had a pitch of $320 \mu \mathrm{m}$. The coupled cladding mode was identified to be the $\mathrm{LP}_{08}$ mode. Two gratings were over-coupled gratings. In an over-coupled grating, the light at the resonance wavelength launched into the core was coupled completely to the cladding at the middle of the grating and then back to the core at the end of the grating. Therefore, the contrast at the resonance wavelength of an over-coupled grating should be close to zero. During the writing process, we monitored the transmission spectrum of the grating and controlled the UV dosage to ensure that the over-coupling condition was achieved. The resonance wavelength and the contrast of the fabricated grating were measured to be $1580.5 \mathrm{~nm}$ and $0.8 \mathrm{~dB}$, respectively. The idea of using over-coupled gratings is to increase the coupling strengths of the gratings, so that a smaller offset distance and hence a more compact sensor configuration can be used.
Figure 4 shows the dependence of the output power of the sensor on the surrounding refractive index for two offset distances, $0 \mathrm{~mm}$ and $5 \mathrm{~mm}$, respectively. As shown in Fig. 4, the output power increases with the surrounding refractive index and the sensitivities are similar for both offset distances. The absolute output power is, however, larger for a longer offset distance.

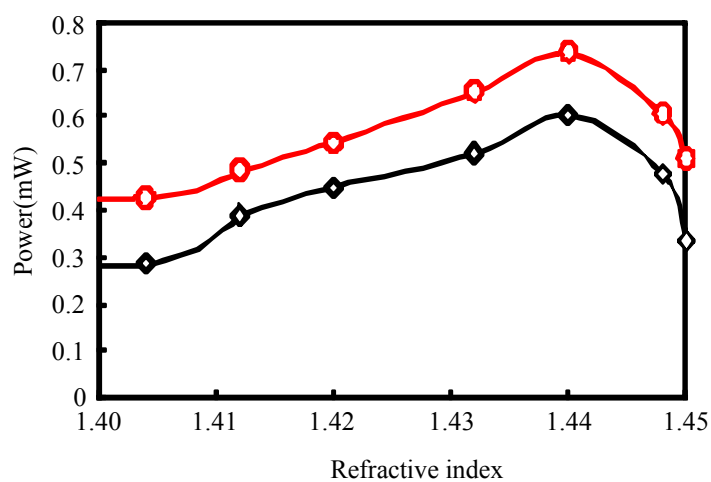

Fig. 4 Dependence of the sensor output on the surrounding refractive index for two parallel over-coupled gratings with an offset distance of $0 \mathrm{~mm}$ (lower curve) or $5 \mathrm{~mm}$ (upper curve).

As shown in Figs. 2 and 4, the output power level for the case of two over-coupled gratings with a zero offset distance is more or less the same as that for the case of two normal gratings with a $10 \mathrm{~mm}$ offset distance. These results confirm that the use of over-coupled gratings does lead to a more compact sensor without compromising the refractive-index sensitivity.

We measured the transmission spectra of two parallel gratings at different surrounding refractive indices and present the results in Fig. 5. The resonance wavelength of the grating coupler shifts towards the shorter wavelength, as the surrounding refractive index increases, which is consistent with previously reported results [12]. Because the response of the photodetector covers a wavelength range over several hundred nanometers and the ASE source has a relatively uniform output spectrum, a shift in the resonance wavelength has little influence on the sensor performance. It is also clear from the transmission spectra that the peak coupling 
efficiency increases with the increasing of the surrounding refractive index. The contrast of the rejection band from the launching fiber decreases with an increase in the surrounding refractive index, which can also be used as a sensor output. The calculated transmission spectra of the sensor are shown in Fig. 6, which agree qualitatively with the experimental results.

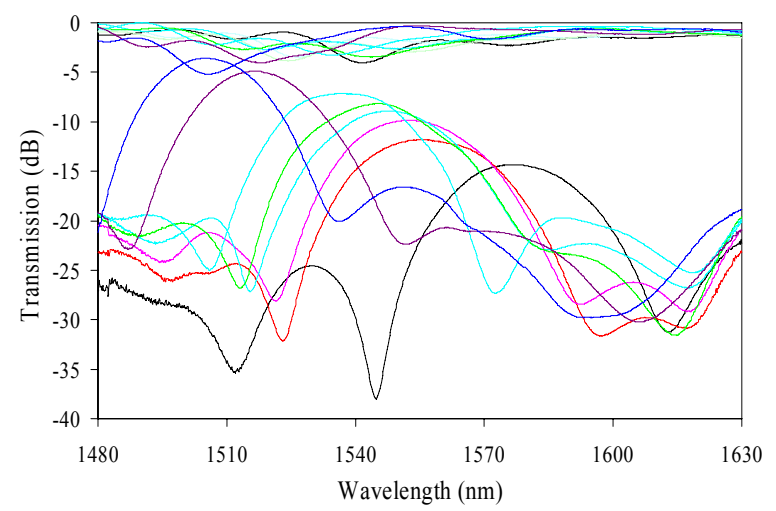

Fig. 5 Transmission spectra from the outputs of two parallel over-coupled LPFGs measured at different surrounding refractive indices: $1.0,1.404,1.412,1.420,1.432,1.440,1.448$, 1.450 (from right to left), showing the band-rejection and band-pass characteristics of the two gratings.

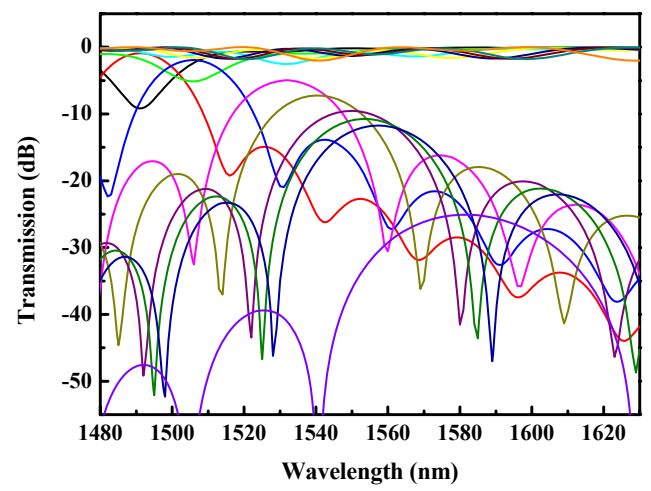

Fig. 6 Transmission spectra from the outputs of two parallel over-coupled LPFGs calculated for different surrounding refractive indices: $1.0,1.404,1.412,1.420,1.432,1.440,1.448$, 1.450 (from right to left), showing the band-rejection and band-pass characteristics of two gratings.

\section{Displacement sensing}

The coupling efficiency between two parallel LPFGs is highly sensitive to the lateral separation between two fibers. Figure 7 shows the calculated power coupling as a function of the fiber separation. In the calculation, we assume that the fiber has a core index of 1.4490 , a core radius of $3.78 \mu \mathrm{m}$, a cladding index of 1.4443, and a cladding radius of $62.4 \mu \mathrm{m}$ and that the gratings are $32 \mathrm{~mm}$ long and have a zero offset distance. As shown in Fig. 7, the output power drops rapidly with the increase of the fiber separation, especially in air. A displacement resolution of the order of a few nanometers (in air) should be possible. The authors are not aware of any other principles based on light-intensity measurements with optical fibers that can provide the same degree of displacement resolution.

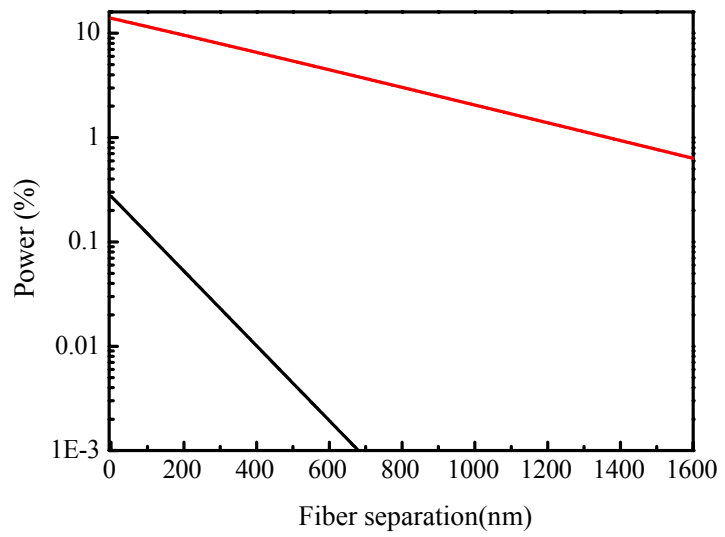

Fig. 7 Calculated power coupling between two parallel LPFGs as a function of the lateral separation between two fibers in air (lower curve) or an index-matching medium with a refractive index of 1.43 (upper curve).

While we are yet to demonstrate a highresolution displacement sensor experimentally by using the configuration of two parallel LPFGs, we present here a proximity sensor based on the same principle. Figure 8 shows the transmission spectra of an over-coupled grating measured before and after a bare fiber placed in proximity to it. It can be seen that the contrast of the grating at the resonance wavelength increases from $1.18 \mathrm{~dB}(23.8 \%)$ to $2.78 \mathrm{~dB}(47.3 \%)$ when the fiber is brought close to the grating. The large drop in the output power (about 23\%) is sufficient to identify the presence of an object within a distance of one micrometer. The use of a laser source emitting at the resonance 
wavelength, instead of the ASE source, can provide a highly sensitive measurement. We can envisage that the same mechanism can be applied to the measurement of minute vibrations, pressure, and strain. What we need is a suitable transducer to convert any physical parameter to be measured into displacement.

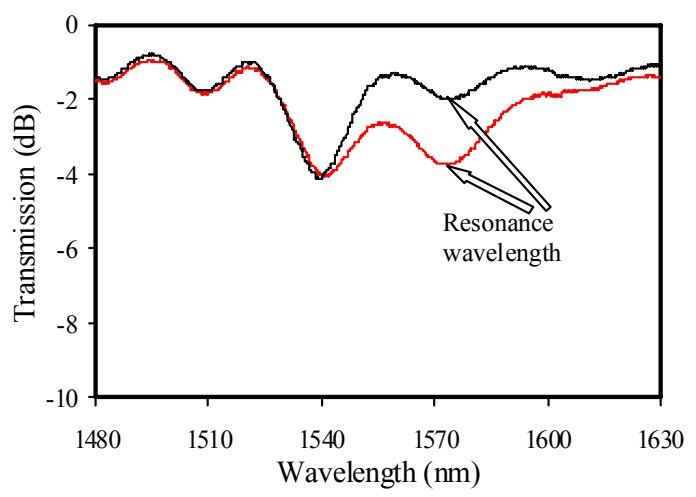

Fig. 8 Transmission spectra of an over-coupled LPFG measured before (upper curve) and after (lower curve) a bare fiber was brought in proximity to it.

\section{Conclusions}

With experiments and simulation results, we have demonstrated a new optical intensity-based sensing mechanism for the measurement of refractive index and minute displacement. This sensing mechanism relies on a change in the amount of light coupled between two parallel LPFGs in response to a change in the surrounding refractive index or the lateral separation between two gratings. The structure of two parallel LPFGs has a potential to be developed into a wide range of low-cost sensors for the measurement of liquid level, chemical concentration, proximity, displacement, vibration, pressure, etc. We should emphasize in particular that this new sensing mechanism can potentially offer a high displacement resolution (a few nanometers) that can usually be achieved with much more complicated sensing schemes. We should also mention the recent demonstrations of a narrow-band coupler formed with an LPFG and a parallel tilted fiber Bragg grating [13] and an LPFG-based fiber ring resonator [14]. These new cladding-mode coupling structures could be explored for the development of a new range of optical fiber sensors.

\section{Acknowledgment}

This research was supported jointly by a grant from the Research Grants Council of the Hong Kong Special Administrative Region, China, under Project CityU 111907, and a research grant from the University of Electronic Science and Technology of China under the Chang Jiang Scholars Program.

Open Access This article is distributed under the terms of the Creative Commons Attribution License which permits any use, distribution, and reproduction in any medium, provided the original author(s) and source are credited.

\section{References}

[1] A. M. Vengsarkar, P. J. Lemaire, J. B. Judkins, V. Bhatia, T. Erdogan, and J. E. Sipe, "Long-period fiber gratings as band-rejection filters," J. Lightwave Technol., vol. 14, no. 1, pp. 58-65, 1996.

[2] K. S. Chiang and Q. Liu, "Long-period gratings for application in optical communications," in Proc. $5^{\text {th }}$ International Conf. on Optical Communications and Networks and $2^{\text {nd }}$ International Symposium on Advances and Trends in Fiber Optics and Applications(ICOCN/ATFO 2006), Chengdu, China, pp.128-133, 2006.

[3] S. W. James and R. P. Tatam, "Optical fibre long-period grating sensors: characteristics and application," Meas. Sci. Technol., vol. 14, no. 5, pp. R49-R61, 2003.

[4] K. S. Chiang, Y. Liu, M. N. Ng, and S. P. Li, "Coupling between two parallel long-period fibre gratings," Electron. Lett., vol. 36, no. 16, pp. 1408-1409, 2000.

[5] K. S. Chiang, F. Y. M. Chan, and M. N. Ng, "Analysis of two parallel long-period fiber gratings," J. Lightwave Technol., vol. 22, no. 5, pp. 1358-1366, 2004.

[6] Y. H. Han, S. B. Lee, C. S. Kim, and M. Y. Jeong, "Tunable optical add-drop multiplexer based on long-period fiber gratings for coarse wavelength division multiplexing systems," Opt. Lett., vol. 31, no. 
6, pp. 703-705 2006.

[7] M. J. Kim, Y. M. Jung, B. H. Kim, W. T. Han, and B. H. Lee, "Ultra-wide bandpass filter based on long-period fiber gratings and the evanescent field coupling between two fibers," Opt. Express, vol. 15, no. 17, pp. 10855-10862, 2007.

[8]Y. Liu, K. S. Chiang, R. J. Rao, Z. L. Ran, and T. Zhu, "Light coupling between two parallel $\mathrm{CO}_{2}$-laser written long-period fiber gratings," Opt. Express, vol. 15, no. 26, pp. 17645-17651, 2007.

[9] Y. Liu and K. S. Chiang, "Broad-band optical coupler based on evanescent-field coupling between three parallel long-period fiber gratings," IEEE Photon. Technol. Lett., vol. 18, no. 1, pp. 229-231, 2006.

[10] Y. Liu, K. S. Chiang, and Q. Liu, "Symmetric $3 \times 3$ optical coupler using three parallel long-period fiber gratings," Opt. Express, vol. 15, no. 10, pp. 64946499, 2007.

[11] K. S. Chiang, "Development of long-period fiber grating coupling devices," Appl. Opt., vol. 48, no. 25, pp. F61-F67, 2009.

[12] K. S. Chiang, Y. Liu, M. N. Ng, and X. Dong, "Analysis of etched long-period fibre grating and its response to external refractive index," Electron. Lett., vol. 36, no. 11, pp. 966-967, 2000.

[13] Y. Liu, Q. Liu, and K. S. Chiang, "Optical coupling between a long-period fiber grating and a parallel tilted fiber Bragg grating," Opt. Lett., vol. 34, no. 11, pp. 1726-1728, 2009.

[14] Z. Wang, K. S. Chiang, and Q. Liu, "Microwave photonic filter based on circulating a cladding mode in a fiber ring resonator," Opt. Lett., vol. 35, no. 5, pp. 769-771, 2010. 